\title{
Experimental characterization of Gaussian quantum-communication channels
}

\author{
James DiGuglielmo, ${ }^{1, *}$ Boris Hage, ${ }^{1}$ Alexander Franzen, ${ }^{1}$ Jaromír Fiurášek, $^{2}$ and Roman Schnabel ${ }^{1}$ \\ ${ }^{1}$ Institut für Gravitationsphysik, Leibniz. Universität Hannover and Max-Planck-Institut für Gravitationsphysik \\ (Albert-Einstein-Institute), Callinstrasse 38, 30167 Hannover, Germany \\ ${ }^{2}$ Department of Optics, Palacky University, 17 listopadu 50, 77200 Olomouc, Czech Republic
}

(Received 5 May 2007; published 23 July 2007)

\begin{abstract}
We present a full experimental characterization of continuous-variable quantum-communication channels established by shared entanglement together with local operations and classical communication. The resulting teleportation channel was fully characterized by measuring all elements of the covariance matrix of the shared two-mode squeezed Gaussian state. From the experimental data we determined the lower bound to the quantum channel capacity, the teleportation fidelity of coherent states, and the logarithmic negativity and purity of the shared state. Additionally, a positive secret key rate was obtained for two of the established channels.
\end{abstract}

DOI: 10.1103/PhysRevA.76.012323

PACS number(s): 03.67.Mn, 03.67.Hk

\section{INTRODUCTION}

Continuous-variable quantum-communication channels have been the subject of both theoretical and experimental research for the past few years [1-11]. Similarly to classicalcommunication channels, quantum-communication channels are characterized by a channel capacity. In contrast to classical-communication channels, the capacity of quantumcommunication channels is distinguished by two different quantities; namely, the classical capacity, which gives the number of classical bits that can be faithfully transmitted per use of the channel, and the quantum capacity, which specifies how many quantum bits can be transmitted per use of the channel $[4,12]$. One example of a quantum-communication channel is a teleportation channel, which is established by a shared entangled state with local operations and classical communication between two distant parties $[13,14]$. Of all the possible entangled states that could be used to establish the quantum channel, Gaussian states are of particular interest due to their well-understood theoretical structure and ability to be easily generated experimentally $[3,8]$. Because these states are characterized by a Gaussian Wigner function, only the second moments collected in the state's covariance matrix $(\mathrm{CM})$ are required in order to completely define the state. Experimentally, this means that only a few tomographic measurements need to be conducted, significantly reducing the effort to measure these states. To date, several groups have conducted experiments only partially measuring the CM [15-17].

This paper presents an experimental study of Gaussian quantum-teleportation channels. The teleportation channels are established by distributing two different classes of entangled Gaussian states illustrated in Fig. 1 over a free-space auxiliary channel to two parties, Alice and Bob, together with local operations and classical communication. In our experiment, every single parameter of the CMs is measured. These channels are then characterized by evaluating the lower bounds to the quantum-channel capacity, the teleportation fidelities of coherent states, and the purities and loga-

\footnotetext{
*James.DiGuglielmo@aei.mpg.de
}

rithmic negativities of the shared entangled states. Additionally, two different entanglement criteria are used-the Simon-Peres-Horodecki criterion and an entanglement witness-to verify that the measured state is in fact entangled. This paper is divided into the following sections. In Sec. II, we present an efficient experimental procedure for measuring the entire CM using only five measurement settings. The technical details of our experiment are described in Sec. III. The experimental implementation of the measurement of the entire covariance matrix is discussed in Sec. IV. The formal definition of a quantum channel as well as the quantities that characterize them are presented in Sec. V. The reconstructed CMs from the experimental data are presented in Sec. VI, and finally Sec. VII contains a discussion of the results.

\section{EXPERIMENTAL MODUS OPERANDI}

\section{A. Preliminary considerations}

In order to obtain complete knowledge of a two-mode Gaussian entangled state, it is sufficient to measure its symmetric positive semidefinite ten-parameter covariance matrix $[16,17]$. In its block form, the CM is given by
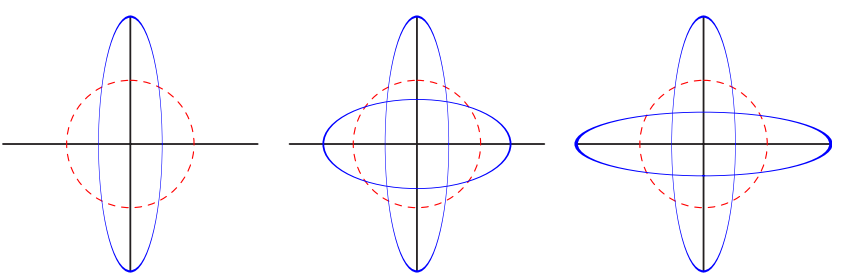

FIG. 1. (Color online) Classes of entanglement: This figure depicts three different classes of entanglement. V-class entanglement (left) is formed by mixing a single-mode squeezed state with the vacuum mode on a balanced beam splitter (BBS). M-class entanglement (center) is formed by mixing two unevenly and oppositely single-mode squeezed beams on a BBS, and S-class entanglement (right) is formed by mixing two equally but oppositely single-mode squeezed beams on a BBS. 


$$
\gamma=\left(\begin{array}{cc}
\mathbf{A} & \mathbf{C} \\
\mathbf{C}^{T} & \mathbf{B}
\end{array}\right)
$$

where $\mathbf{A}, \mathbf{B}$, and $\mathbf{C}$ are $2 \times 2$ matrices which contain the parameters describing Alice's mode, Bob's mode, and the correlations between their modes, respectively. The CM contains the second moments of a state's quadratures, $\gamma_{j k}$ $=\left\langle\Delta r_{j} \Delta r_{k}+\Delta r_{k} \Delta r_{j}\right\rangle$, where $r=\left(x_{A}, p_{A}, x_{B}, p_{B}\right)$ is a vector of quadrature operators and $\Delta r_{j}=r_{j}-\left\langle r_{j}\right\rangle$. We use units such that the covariance matrix of vacuum is equal to the identity matrix. From $\gamma$ can be obtained information regarding entanglement properties of the state (e.g., verification, quantification) as well as the state's purity. In the case of teleportation channels, the lower bound to the quantum-channel capacity and the teleportation fidelity of coherent states can also be obtained from the CM.

For applications such as monitoring of quantumcommunication channels, it is highly desirable to develop techniques such that the reconstruction of a state's CM can be accomplished with the fewest possible measurements. To this end, the structure of the matrix itself can be exploited such that only two measurement settings yield six of the ten independent parameters (simultaneous measurement of the amplitude quadrature of one mode and the phase quadrature of the other mode). Besides these more technical considerations regarding an efficient experimental procedure for the detection and quantification of entangled Gaussian states, there are a number of fundamental issues that must be addressed. These have been elaborated upon by van Enk et al. [18], who gave five criteria that should be met when conducting an entanglement experiment. The heart of the criteria is not to assume too much as to the form, symmetry, or repeatability of the entanglement source for each copy that it produces. The effect of not satisfying these criteria is to increase the risk of overestimating or underestimating the amount of entanglement present in the generated state. Any entanglement verification protocol should satisfy these five criteria. The choice of a verification protocol will ultimately depend on the type of entanglement generated (or thought to have been generated) in an experiment.

The establishment of a quantum-communication channel, such as a teleportation channel, requires the distribution of what van Enk et al. have referred to as a priori entanglement [18]. This type of entanglement is obtained when a source generates many copies of a bipartite state $\rho_{A B}$, such that an entanglement verification protocol can be conducted on a subensemble of them, using the rest to perform a quantuminformation theoretic protocol. A possible verification protocol for a priori entanglement is to perform full tomography on the state. This can be achieved using linear optics and homodyne detection $[19,20]$. This allows for not only a qualitative statement as to whether the state is separable or entangled but also a quantitative statement as to how much. Full tomography is expensive, however, especially when its implementation is solely to obtain information about the channel. As such, it is desirable to develop verification protocols that can be conducted using only partial tomographic measurements while still satisfying the van Enk et al. crite- ria. We now present such a partial tomographic protocol (PTP).

\section{B. Description of the partial tomographic protocol}

The partial tomographic protocol developed to characterize our teleportation channels can be stated as follows.

(1) Alice and Bob simultaneously measure their amplitude and phase quadratures, respectively, while comparing their results by means of classical communication.

(2) Alice and Bob simultaneously measure their phase and amplitude quadratures, respectively, while comparing their results by means of classical communication.

(3) Alice and Bob measure their amplitude quadratures.

(4) Alice and Bob measure their phase quadratures.

(5) Alice and Bob simultaneously measure a linear combination of their amplitude and phase quadratures, respectively.

The fact that every parameter of the $\mathrm{CM}$ is measured prevents one from making an assumption as to the symmetry of the state being measured. Although measuring only the second moments of the state does not give information as to whether the state is Gaussian or not, something which in the strictest sense of the van Enk et al. criteria should not be assumed, an entanglement criterion, such as the Simon criterion, is a sufficient criterion for both Gaussian and nonGaussian states. Furthermore, quantities such as the secret key rate $[21,22]$ or the lower bound to the quantum-channel capacity [8], while indirectly indicating the presence of entanglement, obtain their lower bounds for Gaussian states. As such, one can at worst only underestimate these quantities by measuring just the second moments and assuming that the state is Gaussian.

\section{EXPERIMENTAL SETUP}

In our experiment, we generate the two-mode entangled states by mixing on a balanced beam splitter two squeezed vacuum beams produced by our optical parametric amplifiers (OPAs). The laser source used in our experiment was a continuous-wave nonplanar neodymium-doped yttrium aluminum garnet (Nd:YAG) ring laser with $300 \mathrm{~mW}$ of output power at $1064 \mathrm{~nm}$ and $800 \mathrm{~mW}$ at $532 \mathrm{~nm}$. The latter was used to pump the OPAs to produce two amplitude-squeezed light beams with an approximate power of $0.06 \mathrm{~mW}$ at $1064 \mathrm{~nm}$. Both OPAs were constructed from type-I noncritically phase-matched $\mathrm{MgO}: \mathrm{LiNbO}_{3}$ crystals inside hemilithic cavities. Each cavity was formed by a high-reflection(HR-)coated crystal surface with a reflectivity of $r>0.999$ and a metal-spacer-mounted out-coupling mirror with a reflectivity of $r=0.957$. The intracavity crystal surface was antireflection-coated for both the fundamental $(1064 \mathrm{~nm}, r$ $<0.05 \%)$ and the second harmonic $(532 \mathrm{~nm}, r<0.5 \%)$. The out-coupling mirror had a reflectivity of $r=0.15 \pm 0.02$ for $532 \mathrm{~nm}$. The OPAs were seeded through the HR surface with a coherent laser beam of $15 \mathrm{~mW}$ power and pumped through the out-coupling mirror with various intensities, the lowest being $75 \mathrm{~mW}$, corresponding to a parametric gain of 5 . The length of both OPA cavities as well as the phase of the sec- 


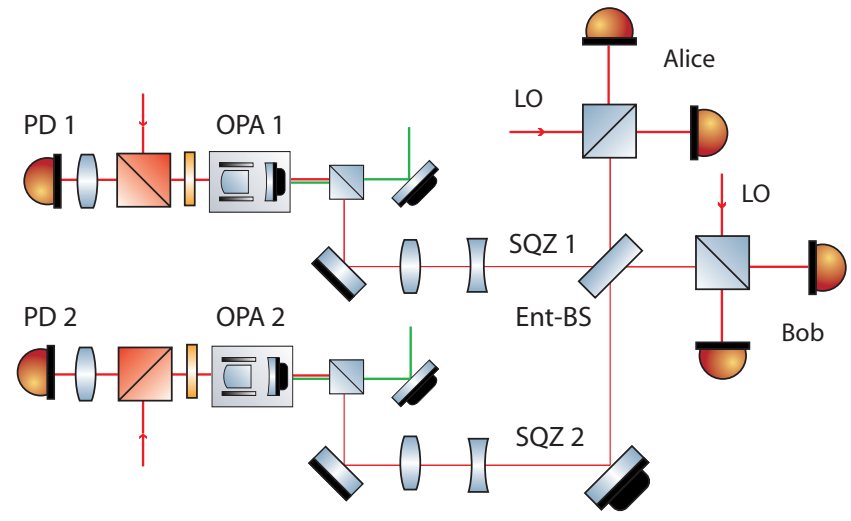

FIG. 2. (Color online) Experimental setup: The squeezed states are generated by the two optical parametric amplifiers (OPAs) and mixed at the entangling beam splitter (Ent-BS). The different classes of entanglement are generated by adjusting the parametric gain setting of the OPAs. The entanglement is then distributed over a free-space channel to the two homodyne detectors Alice and Bob.

ond harmonic were controlled using radio-frequency modulation or demodulation techniques. The error signals were derived from the seed fields reflected from the OPA cavities. A maximum value of $4.0 \mathrm{~dB}$ of nonclassical noise suppression was directly observed using homodyne detection. The shot noise level was defined by mixing the local oscillator with the vacuum mode on a balanced beam splitter and measuring fluctuations of vacuum. The electronic dark noise of the homodyne detectors was approximately $13 \mathrm{~dB}$ below the shot noise level, making dark noise correction of the observed squeezing superfluous. The visibility on both homodyne detectors was $\eta_{\mathrm{vis}}=0.965$, and the quantum efficiency of the photodetectors is estimated to be $\eta_{\text {quantum }}=0.93$, yielding a total detection efficiency of $\eta \approx 0.87$. The phase locks on both the entangling beam splitter and homodyne beam splitters are estimated to be within $3^{\circ}$ of the desired values. The photocurrents produced from the homodyne detectors were first demodulated at a frequency of $7 \mathrm{MHz}$ and lowpass filtered with a corner frequency of $30 \mathrm{kHz}$. They were sampled with a National Instruments sampling card with maximum sampling rate of 1 megasample per second. By independently changing the parametric gain of each amplifier we can generate all three types of entanglement as illustrated in Fig. 1. A diagram of the full experiment is provided in Fig. 2.

\section{EXPERIMENTAL IMPLEMENTATION OF PTP}

Our partial tomographic protocol was implemented using a custom-built data-acquisition system whose software component was developed using LABVIEW and whose hardware component was realized by balanced homodyne detection with external addition and subtraction boxes. The homodyne detectors were designed such that there were multiple outputs of both the dc and ac signals generated by each detector. The ac-subtracted signals from both homodyne detectors were fed simultaneously into the LABVIEW program, where both the variances of the respective electronic channels as well as the covariance of the two electronic channels were calculated in real time. This corresponds to the classicalcommunication component of our protocol. Additional information as to which quadratures produced a given covariance was obtained by recording, in real time, the dc-subtracted signal from one scanned homodyne detector. This was achieved by locking, e.g., Alice's homodyne detector to one quadrature and scanning the phase between the local oscillator and signal beam of Bob's homodyne detector. With this setup, one measurement-e.g., measuring the amplitude quadratures simultaneously-delivered three of the ten required $\mathrm{CM}$ parameters.

There are two main features of our implementation that are noteworthy. First, it allows the manual setting of the measurement basis. The basis information can be obtained by looking at the covariance of the two electronic channels. A zero covariance indicates the measurement of two orthogonal quadratures for a symmetric state. Although both the verification and quantification of entanglement are basis independent, the form of the CM is not. For the case of an optimally entangled Einstein-Podolsky-Rosen (EPR) state, one would expect nonzero parameters for half the elements of the $\mathrm{CM}$ in an orthogonal measurement basis. While the choice of a basis is arbitrary, it must be consistent. Failure to measure every parameter of the CM in the same basis is tantamount to random experimental error. Failure to measure some of the parameters in the same basis is systematic error, as it adds a constant offset to only some of the parameters. The result of these error sources, especially systematic error, is to give a false estimate of quantities of interest such as the logarithmic negativity [23], or to reconstruct a nonphysical state.

Second, systematic error can be reduced. As a result of the real-time evaluation of the covariance between the homodyne detector outputs and the dc-subtracted signal from a scanned homodyne detector, one can determine which quadratures are correlated, anticorrelated, and not correlated. This information helps to reduce the systematic error, because it provides a means by which to adjust the phase angle between the optical local oscillator and signal beam independent of any dc offsets on the error signal. This contributes to the overall consistency of the entanglement detection.

\section{THEORETICAL DESCRIPTION OF QUANTUM-COMMUNICATION CHANNELS}

In order to more deeply understand the equivalence between a shared entangled state and an established quantumcommunication channel, such as for a teleportation channel, it is necessary to understand the theoretical structure of quantum-communication channels. It is also within this framework that these channels obtain their physical meaning. To this end, this section will review the necessary theoretical concepts in order to understand the experimental results of Sec. VI.

A quantum channel is a trace-preserving completely positive map $T$ that transforms quantum states according to $\rho \mapsto T(\rho)$ [7]. They can be understood to originate as the result of a unitary interaction $U$ of a state $\rho$ with the environment described by another Hilbert space $\mathcal{H}_{E}$ which is in a state $\rho_{E}$, 


$$
T(\rho)=\operatorname{Tr}_{E} U\left(\rho \otimes \rho_{E}\right) U^{\dagger},
$$

where $\operatorname{Tr}_{E}$ denotes the partial trace with respect to $\mathcal{H}_{E}$ $[3,7,24]$. An important subclass of these channels are the Gaussian channels, which are characterized by a Gaussian unitary $U$, determined by a quadratic bosonic Hamiltonian, and a Gaussian state $\rho_{E}$ [3]. At the level of covariance matrices, which offer a complete description of Gaussian states and would be measured in all practical applications of continuous-variable quantum-information protocols, the action of a channel is given by

$$
\gamma \mapsto X^{T} \gamma X+Y .
$$

The condition to ensure that the transformation is completely positive is given by

$$
Y+i \Omega-i X^{T} \Omega X \geqslant 0,
$$

where

$$
\Omega=\left(\begin{array}{ll}
\sigma & 0 \\
0 & \sigma
\end{array}\right)
$$

is the symplectic form with

$$
\sigma=\left(\begin{array}{cc}
0 & 1 \\
-1 & 0
\end{array}\right) .
$$

The formula Eq. (4) represents the necessary and sufficient condition for complete positivity of the Gaussian map given by Eq. (3); see, e.g., Refs. [25-29]. It is possible to interpret this condition as the generalized Heisenberg inequality. According to the Jamiolkowski isomorphism [29], every completely positive map is isomorphic to a positive semidefinite operator on the tensor product of Hilbert spaces of input and output states. In the case of Gaussian CP maps this operator becomes an infinitely squeezed Gaussian state characterized by matrices $X$ and $Y$. The generalized Heisenberg inequality for the covariance matrix of this state is equivalent to Eq. (4); cf. Ref. [25].

The usual quantum-information protocols, e.g., teleportation and quantum memory, can all be considered as quantum channels [8]. In this paper, we consider a special subclass of teleportation channels established by means of a shared entangled state together with local operations and classical communication. An important characteristic of teleportation channels, as well as quantum channels in general, is their capacity to transmit quantum information, quantified in units of qubits. To this end the quantum capacity [3,30-32] of an arbitrary channel $T$ is given by

$$
\begin{gathered}
Q(T)=\lim _{n \rightarrow \infty} \frac{1}{n} \sup _{\rho} J\left(\rho, T^{\otimes n}\right), \\
J(\rho, T)=S(T(\rho))-S((T \otimes \mathrm{id})(\psi)),
\end{gathered}
$$

where $\psi$ is a purification of $\rho$ and $J$ is known as the coherent information. The coherent information was first introduced by Schumacher and Nielsen in connection with error correction [33]. With regard to its operational interpretation, the coherent information quantifies the amount of information the environment has obtained about the state transversing it.
Another information theoretic quantity related to the coherent information is the quantum conditional entropy [34-36] defined by

$$
S(B \mid A)=S\left(\rho_{A B}\right)-S\left(\rho_{A}\right),
$$

where $S\left(\rho_{A B}\right)$ and $S\left(\rho_{A}\right)$ stand for the von Neumann entropies of the total state $\rho_{A B}$ and the part of the total state held by Alice, $\rho_{A}$, respectively. The conditional entropy quantifies the amount of quantum information Bob must send to Alice such that she can recreate the total state $\rho_{A B}$, given her prior knowledge of it, as quantified by $S\left(\rho_{A}\right)$. As such, the conditional entropy quantifies Alice's ignorance of the total state. The coherent information Eq. (6) depends on both the channel $T$ as well as on the input state $\rho$ to the channel. In order to evaluate the quantum capacity of an arbitrary channel $T$, the coherent information must be maximized over all possible input states and regularized over many uses of the channel. For teleportation channels, where $T$ would correspond to a shared entangled state with CM $\gamma$; however, a lower bound to the quantum capacity can be obtained by first applying a distillation protocol to the state in order to obtain $k$ maximally entangled pairs of quantum bits (ebits). The teleportation protocol could then be conducted using these ebits. It was shown by Wolf et al. [8] that the number of ebits that can be obtained from a given state with $\mathrm{CM} \gamma$ can be bounded from below by the right-hand side of

$$
Q(T) \geqslant S\left(\gamma_{A}\right)-S(\gamma) \equiv Q_{L},
$$

which in turn gives a lower bound to the quantum-channel capacity. Here $S(\gamma)$ denotes the von Neumann entropy of a Gaussian state with CM $\gamma$. The development of entanglement distillation protocols is an active area of current research. A major step toward implementation of entanglement distillation for continuous variables $[37,38]$ has been made by the demonstration of squeezed-state purification $[39,40]$ and subtraction of single photons from squeezed states [41-43]. As shown in [44], the protocol demonstrated in [39] is quite general and can be extended in a straightforward manner to an iterative purification protocol as well as to entanglement distillation in the presence of non-Gaussian decoherence.

In addition to the quantum capacity, there are a number of other quantities that will contribute to the characterization of our teleportation channels. To begin with, the state condition, defined by

$$
\gamma+i \Omega \geqslant 0,
$$

where $\Omega$ is again the two-mode symplectic form, determines whether the reconstructed $\mathrm{CM}$ corresponds to a physical state [45]. We define $\lambda$ as the minimum eigenvalue of $\gamma+i \Omega$ and the inequality (9) holds if and only if $\lambda \geqslant 0$. In order to verify that the channel has been established using entanglement, the Simon-Peres-Horodecki (Simon) criterion [45] can be used and can be formulated as

$$
\gamma^{T_{A}}+i \Omega \geqslant 0,
$$

where $\gamma^{T_{A}}=\Lambda \gamma \Lambda$ is the $\mathrm{CM}$ of a state partially transposed with respect to Alice's mode, and $\Lambda=\operatorname{diag}(1,-1,1,1)$ corresponds to a local time-reversal operation on Alice's phase 
TABLE I. Channel characteristics for each class of entanglement used to establish a teleportation channel. They are the state condition $\lambda$, the Simon criterion $\lambda^{T_{A}}$, the optimal entanglement witness $\mathcal{W}$, the logarithmic negativity $E_{\mathcal{N}}$, the lower bound to the quantum channel capacity, $Q_{L}$, the teleportation fidelity of coherent states, $\mathcal{F}$, the purity $\mu$, and the secret key rate $K$.

\begin{tabular}{cccccc}
\hline \hline & \multicolumn{2}{c}{ V class } & & \multicolumn{2}{c}{ S class } \\
\cline { 2 - 3 } \cline { 5 - 6 } Characteristic & Gain 5 & Gain 10 & & Gain 5 & Gain 10 \\
\hline$\lambda$ & $0.033 \pm 0.004$ & $0.034 \pm 0.003$ & & $0.063 \pm 0.003$ & $0.175 \pm 0.005$ \\
$\lambda^{T_{A}}$ & $-0.317 \pm 0.004$ & $-0.349 \pm 0.003$ & & $-0.600 \pm 0.001$ & $-0.566 \pm 0.004$ \\
$\mathcal{W}$ & $-0.341 \pm 0.004$ & $-0.383 \pm 0.003$ & & $-0.599 \pm 0.001$ & $-0.566 \pm 0.004$ \\
$E_{\mathcal{N}}$ & $0.602 \pm 0.003$ & $0.700 \pm 0.004$ & & $1.342 \pm 0.005$ & $1.331 \pm 0.009$ \\
$Q_{L}$ & $-0.071 \pm 0.003$ & $-0.059 \pm 0.004$ & & $0.387 \pm 0.005$ & $0.100 \pm 0.009$ \\
$\mathcal{F}$ & $0.586 \pm 0.003$ & $0.597 \pm 0.003$ & & $0.701 \pm 0.003$ & $0.695 \pm 0.005$ \\
$\mu$ & $0.648 \pm 0.002$ & $0.563 \pm 0.001$ & & $0.608 \pm 0.002$ & $0.301 \pm 0.002$ \\
$K$ & & & $0.323 \pm 0.005$ & $0.120 \pm 0.006$ \\
\hline \hline
\end{tabular}

quadrature only. As before, we define $\lambda^{T_{A}}$ as the minimum eigenvalue of $\gamma^{T_{A}}+i \Omega$. If $\lambda^{T_{A}}<0$, then the state is entangled. In addition to the Simon criterion, an optimal entanglement witness $\mathcal{W}$ was determined by solving the corresponding semidefinite program [46]. The amount of entanglement was quantified using the logarithmic negativity [23], defined by

$$
E_{\mathcal{N}}=\log _{2}\left\|\rho^{T_{A}}\right\|,
$$

where the basis 2 sets the units to bits. The teleportation fidelity for coherent states [25] is given by

$$
\mathcal{F}=\frac{2}{\sqrt{\operatorname{det} \mathbf{E}}},
$$

where the matrix $\mathbf{E}$ reads

$$
\mathbf{E}=2 \mathbf{D}+\mathbf{R A} \mathbf{R}^{T}+\mathbf{R C}+\mathbf{C}^{T} \mathbf{R}^{T}+\mathbf{B},
$$

and the matrices $\mathbf{A}, \mathbf{B}, \mathbf{C}$, and $\mathbf{C}^{T}$ are obtained from the $\mathrm{CM}$ given by Eq. (1) with

$$
\mathbf{R}=\left(\begin{array}{cc}
1 & 0 \\
0 & -1
\end{array}\right) .
$$

The purity of the state is defined by $\mu=\operatorname{Tr}\left[\rho_{A B}^{2}\right]$ and for Gaussian states we have

$$
\mu=\frac{1}{\sqrt{\operatorname{det} \gamma}} .
$$

Finally, we evaluate the achievable secret key rate for an entangled-state-based quantum key distribution protocol where Alice and Bob both measure a certain quadrature using local homodyne detection on their parts of the shared two-mode state. From the knowledge of the covariance matrix $\gamma$, a lower bound on the achievable secret key rate can be calculated by assuming that the state is Gaussian and using the formula

$$
K=I_{A B}-\chi(A: E) .
$$

Here, $I_{A B}$ is the classical mutual information between Alice's and Bob's measured data and $\chi(A: E)$ denotes the Holevo bound between Alice and an eavesdropper Eve [21,22]. This latter quantity can be expressed as $\chi(A: E)=S\left(\rho_{A B}\right)-S\left(\rho_{B}^{a}\right)$, where $\rho_{B}^{a}$ is a normalized density matrix of Bob's mode conditional on Alice's measurement outcome $a$. Note that, for Gaussian states and homodyne detection, $S\left(\rho_{B}^{a}\right)$ does not depend on the measurement outcome $a$, which justifies the use of the above expression.

\section{EXPERIMENTAL RESULTS}

The partial tomographic protocol presented in Sec. II B was used to characterize teleportation channels established by two different classes of distributed bipartite entanglement. Data acquisition was performed using a LABVIEW program. One million data points were recorded per measurement setting. The data were then divided into ten separate data blocks each with 100000 points. Covariance matrices were generated from each of the ten and averaged, yielding an average $\mathrm{CM}$. For each CM, the channel characteristics were calculated and averaged. The standard error was then calculated for the $95 \%$ confidence interval. With respect to the CMs, this ranged from \pm 0.001 to \pm 0.01 . The first class, to be known as V-class entanglement, was formed by mixing a single-mode squeezed vacuum state with the vacuum mode on a balanced beam splitter. According to the formalism presented by Wolf et al. [47], this represents the optimal entangling scheme for these input states. This experiment was conducted for parametric gain settings of 5 and 10 . The reconstructed V-class covariance matrix $(\mathrm{CM})$ for the parametric gain 5 setting is given by

$$
\left(\begin{array}{cccc}
0.751 & -0.146 & 0.307 & -0.000 \\
-0.146 & 3.175 & -0.000 & -2.129 \\
0.307 & -0.000 & 0.706 & -0.102 \\
-0.000 & -2.129 & -0.102 & 3.181
\end{array}\right) .
$$

The channel characteristics are presented in Table I. They include in order of appearance: the state condition Eq. (9), the Simon criterion Eq. (10), an optimal witness; the logarithmic negativity Eq. (11), the lower bound to the quantum 

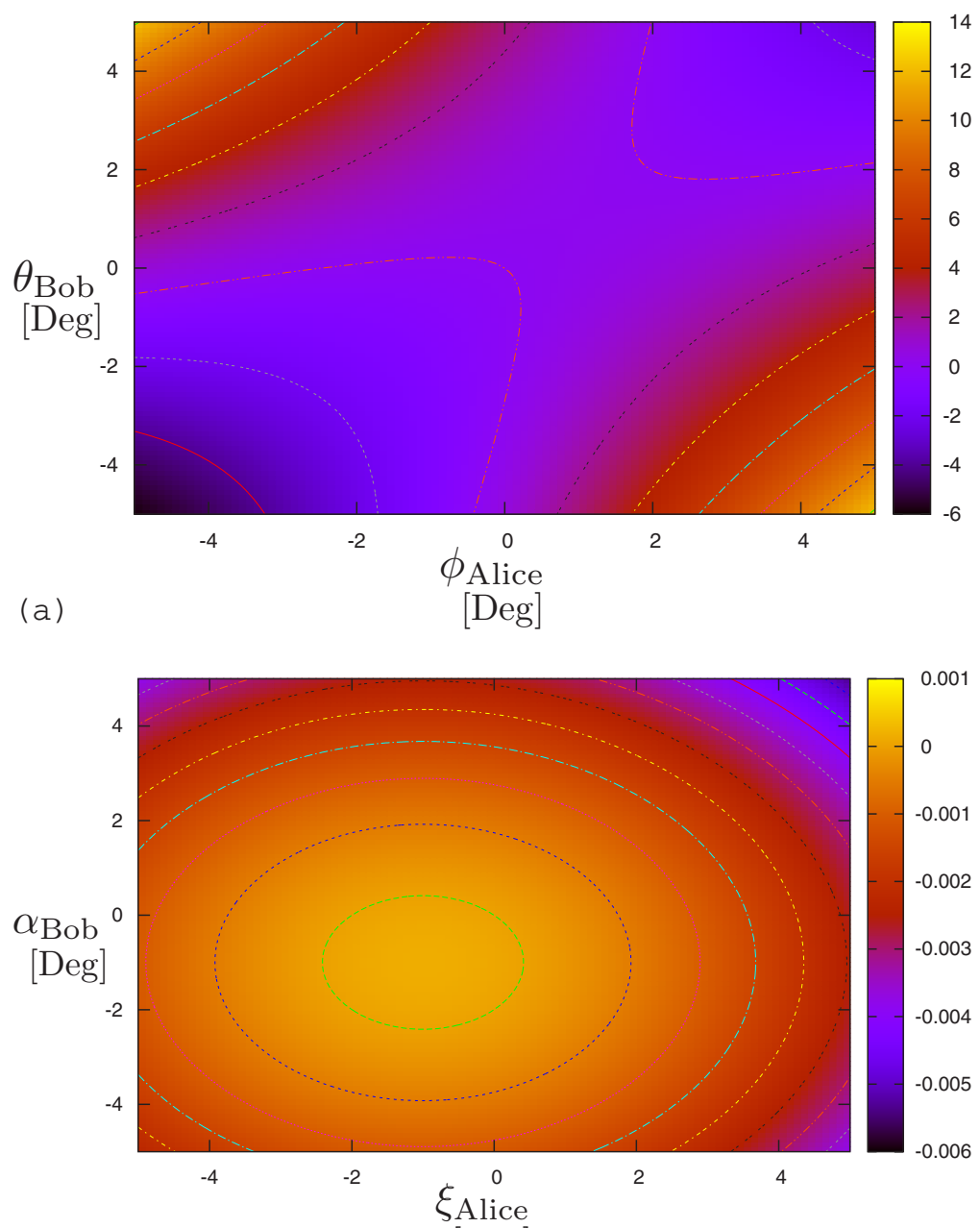

FIG. 3. (Color online) Error analysis: These plots depict the error on the logarithmic negativity for systematic phase offsets on the quadratures (a), and on their linear combination (b). It is seen that the linear combination parameters are more robust to experimental systematic error than the individual quadratures and their dependencies. (b)

[Deg] channel capacity Eq. (8), the teleportation fidelity of coherent states Eq. (12), and the purity of the entangled state Eq. (15). The state condition demonstrates that the reconstructed $\mathrm{CM}$ is a true CM, i.e., that the CM corresponds to a physical state. This serves as an indicator if the measurement has been conducted correctly. Both the Simon criterion and the entanglement witness serve as a check if the state is separable or entangled. The advantage of using an entanglement witness is that it corresponds to the optimized measuring device that can be reconstructed from the measured data [46]. As a result of this optimization, measuring a witness may involve even fewer measurement settings in order to optimally detect the entanglement of the state.

The V-class parametric gain $10 \mathrm{CM}$ reads

$$
\left(\begin{array}{cccc}
0.686 & -0.054 & 0.326 & 0.003 \\
-0.054 & 4.625 & 0.001 & -3.584 \\
0.326 & 0.001 & 0.678 & -0.031 \\
0.003 & -3.584 & -0.031 & 4.681
\end{array}\right)
$$

with the corresponding channel characteristics also given in Table I. In both cases, the lower bound to the channel capacity is negative. The teleportation fidelities $\mathcal{F}$, both being greater than $1 / 2$, indicate the presence of entanglement. The negative values for the Simon criterion and entanglement witness clearly show that the measured state was entangled.

The second class of entanglement generated, to be known as S-class entanglement, was established by mixing two equally but oppositely squeezed beams on a balanced beam splitter. For ideal pure squeezed states, this would yield the two-mode squeezed vacuum state. The reconstructed S-class CMs for the parametric gain 5 and 10 settings read

$$
\left(\begin{array}{cccc}
2.359 & 0.132 & 1.885 & 0.028 \\
0.132 & 2.205 & 0.008 & -1.883 \\
1.885 & 0.008 & 2.266 & 0.372 \\
0.028 & -1.883 & 0.372 & 2.427
\end{array}\right)
$$

and

$$
\left(\begin{array}{cccc}
4.200 & -0.090 & 3.773 & -0.033 \\
-0.090 & 4.462 & 0.035 & -4.216 \\
3.773 & 0.035 & 4.228 & -0.208 \\
-0.033 & -4.216 & -0.208 & 4.842
\end{array}\right)
$$

respectively. The corresponding channel characteristics are listed in the third and fourth columns of Table I, respectively. Comparing the two logarithmic negativities of the V-class 


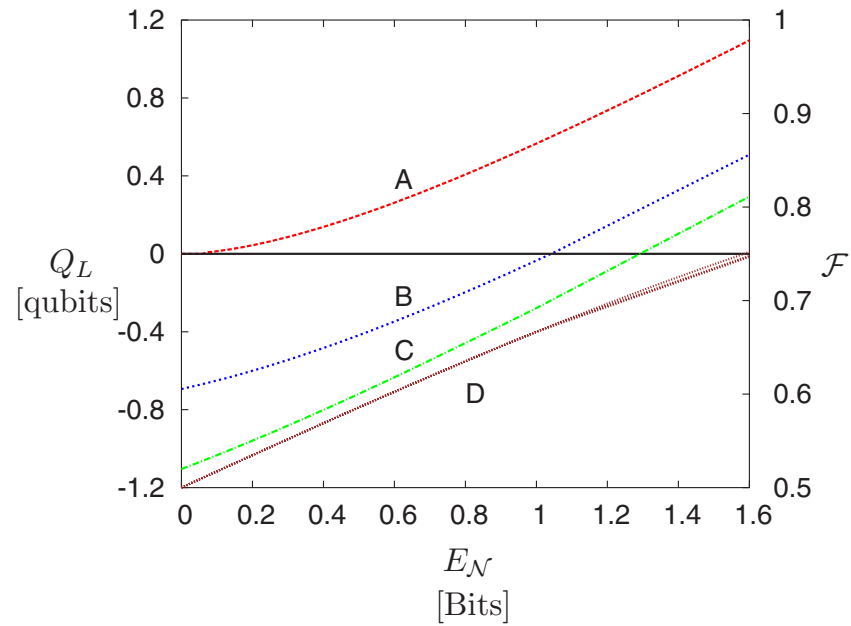

FIG. 4. (Color online) S-class entanglement: This plot depicts the dependence of the lower bound to the quantum-channel capacity, $Q_{L}$, on the purity of the entangled state, where curve $A$ is for $\mu=1$, curve $B$ for $\mu=0.5$, and curve $C$ for $\mu=0.2$, and the amount of entanglement. Interesting for the application of a state-merging protocol or to obtain a positive secret key rate is the point at which $Q_{L}$ becomes positive. It is seen that, as the purity of the state decreases, more entanglement is required for it to become positive. Curve $D$ is the fidelity for each case.

and S-class entangled states, it is seen that the introduction of another squeezed beam increases the amount of entanglement for the same gain setting by roughly a factor of 2 . The lower bound to the quantum capacity also now shows a positive value for each $\mathrm{S}$-class state. The sign of $Q_{L}$ is dependent on both the purity of the state and the amount of entanglement, a relationship that will be explored more fully in Sec. VII. The fidelities are both greater than 0.5 , indicating the presence of entanglement. The fidelity of the gain $5 \mathrm{~S}$-class state, $\mathcal{F}=0.701$, breaks the $2 / 3$ no-cloning limit, which is experimentally significant $[48,49]$. The difference of purities can be understood when we consider that, for V-class entanglement, the vacuum mode introduces only a fixed amount of noise, whereas, for S-class entanglement, the extra noise introduced into the entangled state in the form of antisqueezing is not fundamentally bounded.

As a final result, a positive secret key rate was obtained from both S-class states. With a resolution bandwidth of $50 \mathrm{kHz}, 16.1 \mathrm{kbit} / \mathrm{s}$ of a secure key could be extracted using the channel established by the parametric gain 5 setting. This shows that the S-class entangled states can be used for continuous-variable quantum cryptography. Additionally, drawing on the recent results of Horodecki et al. [35,36], the positive $Q_{L}$ 's indicate that state merging can be achieved using only local operations and classical communication.

\section{DISCUSSION}

It is seen that nonzero entries appear in reconstructed CMs where zero normally would have been expected. The question remains: At what point do these nonzero entries become significant? An analysis of the error incurred as a result of either falsely measuring, or not measuring at all, the
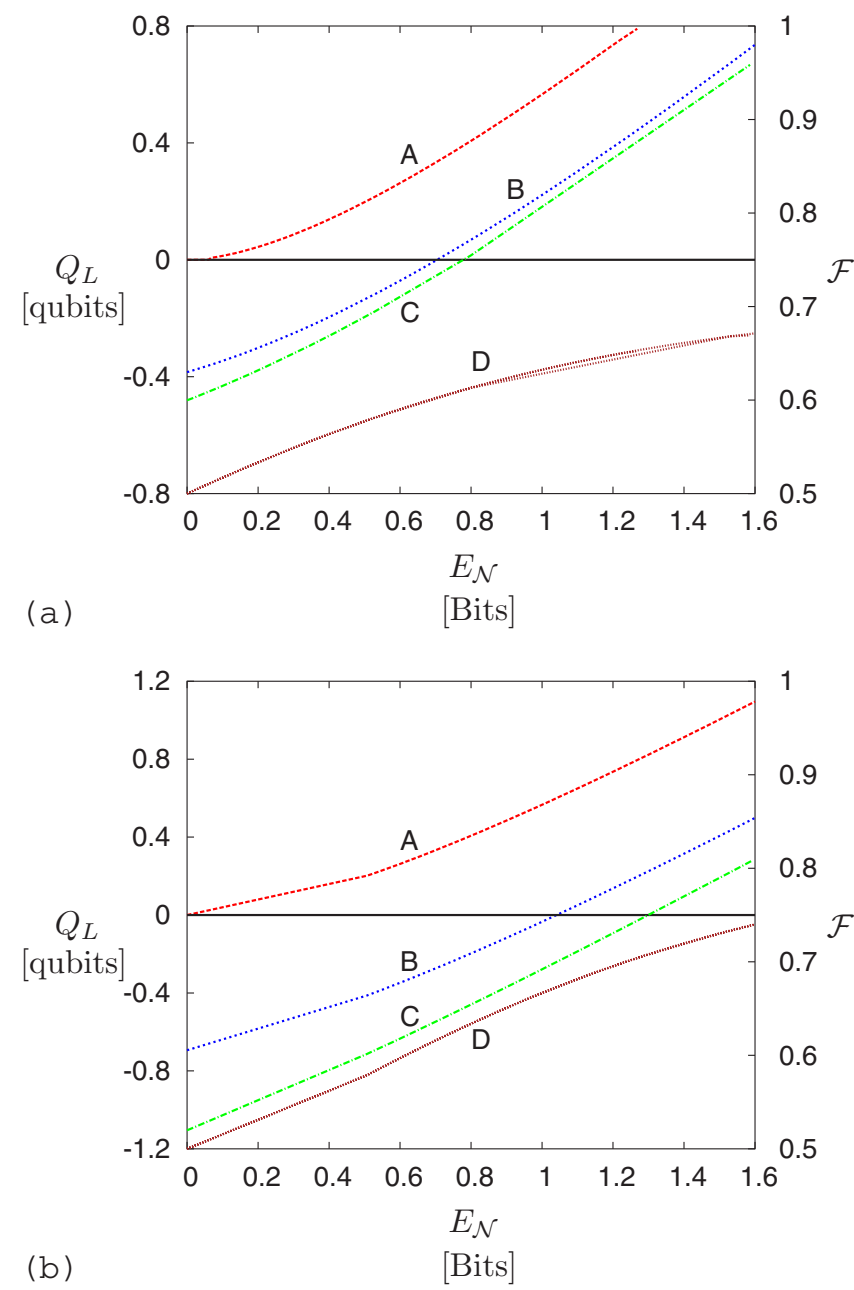

FIG. 5. (Color online) V-class (a) and M-class (b) entanglement: These plots were created in a similar fashion as Fig. 4, but for the $\mathrm{V}$-class and M-class entanglement. As in the previous plot, curve $A$ corresponds to a pure state, $\mu=1$, curve $B$ to $\mu=0.5$, and curve $C$ to $\mu=0.2$. In both plots, the lower bound to the quantum-channel capacity, $Q_{L}$, becomes positive before the $\mathrm{S}$-class entanglement for the same purity.

nonstandard entries has been conducted in [17] and a similar analysis is presented here. In Fig. 3 is illustrated the percent error on the logarithmic negativity in the presence of phase offsets on the homodyne detectors. Two cases are numerically investigated. Figure 3(a) is the effect of phase offsets on measuring the amplitude and phase quadratures. The effect of phase offsets on measurements of the linear combination of the quadratures, assuming the individual quadratures have been properly measured, is illustrated in Fig. 3(b). The simulation was conducted by generating a covariance matrix corresponding to a pure nonoptimally entangled $\mathrm{S}$-class state, whose quadrature variances were dependent on four independent parameters, namely, $\phi_{\text {Alice }}, \theta_{\text {Bob }}, \xi_{\text {Alice }}$, and $\alpha_{\text {Bob. }}$. The parameters $\phi$ and $\theta$ correspond to the independent phase offsets applied to Alice's and Bob's quadratures, respectively. The parameters $\xi$ and $\alpha$ correspond to the independent phase offsets applied to Alice's and Bob's linear combination of quadratures. In this way, the effect of incor- 
rectly measuring the quadratures on the value of the covariance between them can be analyzed. In Fig. 3(a) the percent error on the logarithmic negativity dependent on phase offsets on Alice's and Bob's quadratures is presented. It is seen that, in the region of $\phi, \theta \in[-2,2]$, the percent error can be as high as $-2 \%$, indicating that the amount of entanglement is underestimated. As the phase offsets increase, so does the error reaching as high as $14 \%$ overestimation and $-6 \%$ underestimation. The percent error dependent on phase offsets on the linear combination terms is presented in Fig. 3(b). It is seen that the error is significantly less for the same region as in the quadrature case. This illustrates that the linear combination terms are far more robust to systematic error than the quadrature terms and their dependencies.

The experimental results highlight a relationship between the purity, logarithmic negativity, and the lower bound to the quantum-channel capacity, $Q_{L}$. This relationship is made explicit in the numerical results presented in Figs. 4 and 5. The $Q_{L}$ for S-class entanglement is shown for three different purities in Fig. 4. It is seen that for pure states i.e., $\mu=1$, the presence of entanglement ensures a positive $Q_{L}$. As the purity of the state decreases, the zero crossing is shifted toward higher levels of entanglement. The teleportation fidelity, plotted on the second abscissa, is independent of the purity of the state (assuming that the channel has been properly homodyned). The purity dependence of the $Q_{L}$ can be further investigated by looking at its behavior for two other classes of entanglement. The $Q_{L}$ for V-class entanglement and for M-class entanglement, formed by mixing two unequally and oppositely squeezed beams on a balanced beam splitter, is shown in Fig. 5. It is readily seen that the zero crossing for less than pure states occurs at lower levels of entanglement than for S-class entanglement. Indeed, the numerical results for M-class entanglement manifest optimal behavior for less than pure states, being positive earlier than for S-class entanglement for the same purity. Although all three entangled states are bipartite Gaussian states, their utility is very much dependent on their underlying construction.

\section{CONCLUSION}

In this work, we have presented an efficient method for the characterization of Gaussian communication channels with which the entire covariance matrix was measured. This method was applied to two different classes of continuousvariable entangled states which were used to establish a teleportation channel between distant parties. The lower bound to the quantum-channel capacity as well as other characteristics of the channel were evaluated from the reconstructed covariance matrix. The relationship between the purity, entanglement class, and quantum-channel capacity was explored numerically. Two of the established teleportation channels delivered both a positive $Q_{L}$ as well as a positive secret key rate.

\section{ACKNOWLEDGMENTS}

We acknowledge financial support from the Deutsche Forschungsgemeinschaft (DFG), Project No. SCHN 757/2-1. J.F. acknowledges financial support from the Ministry of Education of the Czech Republic under the projects Centre for Modern Optics (Grant No. LC06007) and Measurement and Information in Optics (Grant No. MSM6198959213) and from the EU under project COVAQIAL (Grant No. FP6511004). We also thank Paul Cochrane for fruitful conversations and for reading the manuscript prior to publication.
[1] C. M. Caves and P. D. Drummond, Rev. Mod. Phys. 66, 481 (1994).

[2] N. J. Cerf, J. Clavareau, C. Macchiavello, and J. Roland, Phys. Rev. A 72, 042330 (2005).

[3] J. Eisert and M. M. Wolf, arXiv:quant-ph/0505151.

[4] V. Giovannetti, S. Lloyd, L. Maccone, and P. W. Shor, Phys. Rev. A 68, 062323 (2003).

[5] P. Hausladen, R. Jozsa, B. Schumacher, M. Westmoreland, and W. K. Wootters, Phys. Rev. A 54, 1869 (1996).

[6] B. Schumacher and M. D. Westmoreland, Phys. Rev. A 56, 131 (1997).

[7] A. S. Holevo and R. F. Werner, Phys. Rev. A 63, 032312 (2001).

[8] M. M. Wolf, D. Perez-Garcia, and G. Giedke, Phys. Rev. Lett. 98, 130501 (2007).

[9] R. Alléaume, F. Treussart, G. Messin, Y. Dumeige, J. F. Roch, A. Beveratos, R. Brouri-Tualle, J. P. Poizat, and P. Grangier, New J. Phys. 6, 92 (2004).

[10] F. Grosshans, G. V. Assche, J. Wenger, R. Brouri, N. J. Cerf, and P. Grangier, Nature (London) 421, 238 (2003).

[11] R. Schnabel, W. P. Bowen, N. Treps, T. C. Ralph, Hans-A. Bachor, and P. K. Lam, Phys. Rev. A 67, 012316 (2003).
[12] M. Nielsen and I. L. Chuang, Quantum Computation and Quantum Information (Cambridge University Press, Cambridge, UK, 2000).

[13] S. L. Braunstein and H. J. Kimble, Phys. Rev. Lett. 80, 869 (1998).

[14] W. P. Bowen, N. Treps, B. C. Buchler, R. Schnabel, T. C. Ralph, Hans-A. Bachor, T. Symul, and P. K. Lam, Phys. Rev. A 67, 032302 (2003).

[15] J. Wenger, A. Ourjoumtsev, R. Tualle-Brouri, and P. Grangier, Eur. Phys. J. D 32, 391 (2005).

[16] W. P. Bowen, R. Schnabel, P. K. Lam, and T. C. Ralph, Phys. Rev. A 69, 012304 (2004).

[17] J. Laurat, G. Keller, J. Oliveiria-Huguenin, C. Fabre, T. Coudreau, A. Serafini, G. Adesso, and F. Illuminati, J. Opt. B: Quantum Semiclassical Opt. 7, S577 (2005).

[18] S. J. van Enk, N. Lutkenhaus, and H. J. Kimble, Phys. Rev. A 75, 052318 (2007).

[19] G. Breitenbach and S. Schiller, J. Mod. Opt. 44, 2207 (1997).

[20] U. Leonhardt, Measuring the Quantum State of Light (Cambridge University Press, Cambridge, UK, 1997).

[21] R. García-Patrón and N. J. Cerf, Phys. Rev. Lett. 97, 190503 (2006). 
[22] M. Navascués, F. Grosshans, and A. Acín, Phys. Rev. Lett. 97, 190502 (2006).

[23] G. Vidal and R. F. Werner, Phys. Rev. A 65, 032314 (2002).

[24] D. Bruss and G. Leuchs, Lectures on Quantum Information (Wiley-VCH, Berlin, 2006).

[25] J. Fiurášek, Phys. Rev. A 66, 012304 (2002).

[26] B. Demoen, O. Vanheuverzwijn, and A. Verbeure, Lett. Math. Phys. 2, 161 (1977).

[27] G. Lindblad, J. Phys. A 33, 5059 (2000).

[28] J. Eisert and M. B. Plenio, Phys. Rev. Lett. 89, 097901 (2002).

[29] A. Jamiolkowski, Rep. Math. Phys. 3, 275 (1972).

[30] P. W. Shor (unpublished).

[31] I. Devetak, IEEE Trans. Inf. Theory 51, 44 (2005).

[32] S. Lloyd, Phys. Rev. A 55, 1613 (1997).

[33] B. Schumacher and M. A. Nielsen, Phys. Rev. A 54, 2629 (1996).

[34] D. Slepian and J. K. Wolf, IEEE Trans. Inf. Theory 19, 461 (1972).

[35] M. Horodecki, J. Oppenheim, and A. Winter, Nature (London) 436, 673 (2005).

[36] M. Horodecki, J. Oppenheim, and A. Winter, Commun. Math. Phys. 269, 107 (2006).

[37] D. E. Browne, J. Eisert, S. Scheel, and M. B. Plenio, Phys. Rev. A 67, 062320 (2003).
[38] J. Eisert, D. E. Browne, S. Scheel, and M. B. Plenio, Ann. Phys. 311, 431 (2004).

[39] A. Franzen, B. Hage, J. DiGuglielmo, J. Fiurášek, and R. Schnabel, Phys. Rev. Lett. 97, 150505 (2006).

[40] J. Heersink, C. Marquardt, R. Dong, R. Filip, S. Lorenz, G. Leuchs, and U. L. Andersen, Phys. Rev. Lett. 96, 253601 (2006).

[41] A. Ourjoumtsev, R. Tualle-Brouri, J. Laurat, and P. Grangier, Science 312, 83 (2006).

[42] J. S. Neergaard-Nielsen, B. M. Nielsen, C. Hettich, K. Mølmer, and E. S. Polzik, Phys. Rev. Lett. 97, 083604 (2006).

[43] K. Wakui, H. Takahashi, A. Furusawa, and M. Sasaki, arXiv:quant-ph/0609153.

[44] J. Fiurášek, P. Marek, R. Filip, and R. Schnabel, Phys. Rev. A 75, 050302(R) (2007).

[45] R. Simon, Phys. Rev. Lett. 84, 2726 (2000).

[46] P. Hyllus and J. Eisert, New J. Phys. 8, 51 (2006).

[47] M. M. Wolf, J. Eisert, and M. B. Plenio, Phys. Rev. Lett. 90, 047904 (2003).

[48] F. Grosshans and P. Grangier, Phys. Rev. A 64, 010301(R) (2001).

[49] W. P. Bowen, N. Treps, B. Buchler, R. Schnabel, T. C. Ralph, T. Symul, and P. K. Lam, IEEE J. Quantum Electron. 9, 1519 (2003). 